\title{
A Note on Wage Differentials, Fixed-Wages and Adverse Selection
}

\author{
G. Carlier \\ D. Gaumont
}

October 2, 2002

\begin{abstract}
Using $^{1}$ a substitution property of worker's types (productivity and time preference), we propose an explanation for both fixed-wages and wage differentials. Fixed-wages result in bunching at the optimum. Equally productive workers with different time preference accept different wages ${ }^{2}$.
\end{abstract}

Keywords: Wage differentials, Fixed-wages, Labor Contracts, Adverse Selection, Heterogeneous Time Preference.

J.E.L. Classification: D82, J31.

\footnotetext{
${ }^{1}$ G. Carlier, Université Bordeaux 4, GRAPE UMR CNRS 5113, Tel: 00.33.5.56.84.54.73, Fax: 00.33.5.56.84.29.64, e-mail: carlier@montesquieu.u-bordeaux.fr. Corresponding author : D. Gaumont, Université Paris 2, ERMES UMR CNRS 7017, Tel / Fax: 00.33.1.45.87.39.43, e-mail: damien.gaumont@free.fr

${ }^{2}$ We thank an anonymous referee for her/his fruitful suggestions for improving this work. Usual disclaimers apply.
} 


\section{Introduction}

This note is an attempt to explain, in an adverse selection framework, why workers of different productivity may receive the same wage (fixed-wages) and why equally productive workers may receive different wages (wage differentials).

Fixed-wage theory points out that workers having the same wage do not necessarily have the same productivity, Aziariadis (1981), Grossman (1981), Polemarchakis and Weiss (1978), Killingsworth (1988, p. 61-65) and many empirical studies have tested the theory of fixed-wage at the microeconomic level, Abowd and Ashenfelter (1981), Ham (1982). Roughly speaking, this line of empirical research emphasizes the fact that wages are imperfectly correlated with productivities and, when workers' heterogeneity is not reduced to productivity heterogenity, wages vary less than productivities.

Wage-differentials literature emphasizes that wage differences still remain partially unexplained. Empirical tests solely based on workers' productivity fail to explain all the existing variance of wages. Starting from the seminal work of Abowd and Ashenfelter (1981), Kruegger and Summers (1988), many empirical studies have shown that heterogeneity of productivity is far from capturing heterogeneity of wages among agents.

This paper proposes a possible explanation to the empirical observation that wage differentials do not fully capture productivity differentials. That is, workers of different productivity appear to accept contracts that rather than reflect their differences, pool those differences to some degree. A possible reason for this is that there are some costs involved in writing contracts that screen workers more finely. Another answer, the one we develop here, is that there are other dimensions or characteristics than ability which affect the value of the contract to the workers.

In our model, we consider a situation in which workers' type can vary according to two dimensions: productivity and time preference. Time preference is a relevant individual characteristic for the employer since it affects labor supply; time preference captures substitution between leisure today and consumption tomorrow. Less productive workers may compensate their low productivity by a longer effective working time, whereas most productive workers may supply a shorter effective working time. We show, in a particular case, that the firm may pool on the same labor contract workers of varying types who appear to be perfect substitutes for production. Using this substitution property, we develop a particular model in which the two-dimensional adverse selection problem collapses into a one-dimensional one. More precisely we prove that some combination of productivity and time preference sums up all relevant private information. Therefore the firm designs con- 
tract based upon this reduced parameter. Our next result is that bunching always occurs at the optimum for this reduced problem. To summarize, at a first stage, our model provides an explanation for both wage differentials and fixed-wages effects via the substitution property, and, at a second stage, fixed-wages result in bunching at the optimum.

In Section 2, the model is presented. Section 3 is devoted to the one-dimensional reformulation of the problem. Section 4 studies the optimal contract. Finally, Section 5 concludes.

\section{Description of the model}

We consider a two-period model. In period 1, the firm produces a single good.

Consumers work in period 1 and are retired in period 2. At each period $i$, agents consume a single good denoted $C_{i}, i=1,2$. We assume that prices of $C_{1}, C_{2}$ are exogenous and equal to 1.

\subsection{The agent}

Each agent is denoted by his individual characteristics, i.e. a pair of parameters $(\theta, \delta)$ that he perfectly knows, where $\theta$ represents his productivty and $\delta$ his time preference. During the working period, a $(\theta, \delta)$-type agent produces $l$ units of observable output at the real wage $w$, consumes

$C_{1}$ and saves $w-C_{1}$. During the retirement period, each agent consumes $C_{2}$ equal to the discounted return of his first period saving.

A $(\theta, \delta)$-type agent is characterized by a separable intertemporal utility function:

$$
U\left(C_{1}, C_{2}, \delta, t\right)=v_{1}\left(C_{1}\right)+t+\delta v_{2}\left(C_{2}\right)
$$

where $\left(v_{1}, v_{2}\right)$ are strictly concave increasing functions, $t$ represents leisure time in period 1. Let $T$ be the total amount of time available in period 1 . An agent of productivity $\theta$ who produces $l$ units of output, has leisure time $t$ :

$$
t=T-l / \theta .
$$

Given wage $w$, agent chooses consumptions $\left(C_{1}, C_{2}\right)$ as solutions of

$$
\max _{\left(C_{1}, C_{2}\right)} U\left(C_{1}, C_{2}, \delta, t\right) \text { s.t. } 0 \leq C_{1} \leq w, 0 \leq C_{2} \leq(1+r)\left(w-C_{1}\right),
$$

where $r$ is the exogenous interest rate. Denote by $\left(C_{1}^{*}(\delta, w), C_{2}^{*}(\delta, w)\right)$ the solutions of this program. The indirect utility of $(\theta, \delta)$ agents is:

$$
\tilde{V}(w, l, \theta, \delta)=v_{1}\left(C_{1}^{*}(\delta, w)\right)+T-l / \theta+\delta v_{2}\left(C_{2}^{*}(\delta, w)\right) .
$$


Our aim is to study optimal incentive-compatible labor contracts. At this level of generality, agents' preferences are given by (3) so that we have a technically difficult twodimensional screening problem. It cannot be solved in general, we therefore focus on the particular tractable case where $v_{1}=0$, hence we assume ${ }^{3}$ :

Assumption 1 Consumption only takes place at period $2, v_{1}=0$ so that $C_{2}^{*}(\delta, w)=$ $(1+r) w$. Period 2 utility $v_{2}$, relabeled $v$, is strictly concave increasing and nonnegative.

\subsection{The firm}

The firm sets labor contracts in order to hire a continuum of heterogeneous agents whose $(\theta, \delta)$ are unobservable. In the remainder of the paper, the firm knows the distribution of types which is characterized by a density function $f(.,$.$) that is assumed to be of class C^{1}$, and strictly positive on the rectangle $\Omega:=[\underline{\theta}, \bar{\theta}] \times[\underline{\delta}, \bar{\delta}] \subset(0,+\infty)^{2}$.

A labor contract is a direct mechanism which specifies the wage function $w$ and the individual production function $l$, with respect to the agent's type. The problem of the principal consists in finding incentive-compatible contracts that maximize its profit.

\section{Reformulation of the problem}

In this section, we reduce the asymmetry of information to one dimension. To that end, we use full substitution between the two initial parameters. Incentive-compatibility implies that very heterogeneous agents get the same contract : very skilled workers with low time preference and less productive workers with high time preference group on the same contract. In other words, the two parameters may compensate each other.

\subsection{Incentive-compatible labor contracts}

This subsection specifies the relevant one-dimensional parameter on which the firm sets labor contracts. Using Assumption 1 in (3) we get

$$
\tilde{V}(w, l, \theta, \delta)=T-l / \theta+\delta v((1+r) w)
$$

Since factor $1+r$ plays no role, we shall omit it. Using an increasing affine transformation, preferences become:

$$
\theta \delta v(w)-l
$$

\footnotetext{
${ }^{3}$ In fact, the problem is also tractable when $v_{1}=v_{2}$ is a CARA function, this case will be shortly discussed in the conclusion, we refer to Carlier and Gaumont (2000) for details.
} 
Definition 1 A labor contract is a pair of nonnegative functions $(\theta, \delta) \mapsto(w(\theta, \delta), l(\theta, \delta))$.

$A$ contract $(w, l)$ is feasible if and only if it is incentive-compatible and individually rational, i.e. it satisfies, for all $\left((\theta, \delta),\left(\theta^{\prime}, \delta^{\prime}\right)\right) \in \Omega \times \Omega$ :

$$
\begin{gathered}
\theta \delta v(w(\theta, \delta))-l(\theta, \delta) \geq \theta \delta v\left(w\left(\theta^{\prime}, \delta^{\prime}\right)\right)-l\left(\theta^{\prime}, \delta^{\prime}\right) \\
\theta \delta v(w(\theta, \delta))-l(\theta, \delta) \geq v_{0}
\end{gathered}
$$

where $v_{0} \geq 0$ is the reservation utility ${ }^{4}$.

It is clear that incentive-compatibility (5) implies that feasible contracts only depend on the unidimensional new parameter $\alpha=\theta \delta$. Therefore this parameter is an exhaustive statistic which sums up all relevant information for the firm. Such an unidimensional exhaustive statistic captures substitution between productivity and time preference. Indeed, at each specific level of this parameter, different individuals group on the same contract, explaining fixed-wages. Moreover, at a given level of productivity, identical productive individuals with different time preference receive different wages, explaning wage differentials. More precisely, feasible contracts are fully characterized by:

Proposition 1 A contract $(w, l)$ is feasible if and only if

- $(w, l)$ is $\alpha$-dependent: $(w, l)=(w(\alpha), l(\alpha))$,

- $w$ is non-decreasing,

- $v(w(\alpha))=V^{\prime}(\alpha)$ a.e. where

$$
V(\alpha):=\alpha v(w(\alpha))-l(\alpha)
$$

Since it is fairly standard in unidimensional adverse selection problems, the proof is left to the reader. Note that the wage $w$ and the production $l$ are non-decreasing with respect to $\alpha$, and the utility $V$ is convex and non-decreasing since $v$ is nonnegative.

\footnotetext{
${ }^{4}$ Here, the fact that $v_{0}$ is not type dependent (which considerably simplifies the analysis) can be justified in the following way: assume that unemployed workers receive the null contract $w=0, l=0$ and that $v(0)=$ 0 , in this case the constraint that employed workers with contract $(w, l)$ are better-off than unemployed agents is equivalent to $(6)$ with $v_{0}=0$. More generally one should consider participation constraints of the form : $\theta \delta v(w(\theta, \delta))-l(\theta, \delta) \geq \theta \delta v\left(w_{0}\right)-l_{0}$.
} 


\subsection{The problem of the firm}

The program of the firm is to maximize its expected profit in the set of admissible labor contracts:

$$
\max _{(w, l) \text { feasible }} \int_{\Omega}(l(\theta, \delta)-w(\theta, \delta)) f(\theta, \delta) d \theta d \delta .
$$

Since by Proposition $1(w, l)$ depends on $\alpha$ we can rewrite

$$
\Pi=\int_{\Omega}(l(\theta, \delta)-w(\theta, \delta)) f(\theta, \delta) d \theta d \delta=\int_{\underline{\alpha}}^{\bar{\alpha}}(l(\alpha)-w(\alpha)) h(\alpha) d \alpha
$$

where $\underline{\alpha}:=\underline{\theta \delta}, \bar{\alpha}:=\overline{\theta \delta}$ and $h$ is the density function of $\alpha$ (see computations and properties of $h$ in Appendix). Firstly, by Proposition 1

$$
v(w(\alpha))=V^{\prime}(\alpha) \text { with } l(\alpha):=\alpha v(w(\alpha))-V(\alpha)
$$

secondly it is clear that individual rationality (6) binds at $\alpha=\underline{\alpha}: V(\underline{\alpha})=v_{0}$ so that

$$
l(\alpha)=\alpha v(w(\alpha))-v_{0}-\int_{\underline{\alpha}}^{\alpha} v(w(s)) d s
$$

replacing in (7) and integrating by parts, we obtain

$$
\Pi=\Pi(w)=\int_{\underline{\alpha}}^{\bar{\alpha}}[v(w(\alpha))(\alpha h(\alpha)+H(\alpha)-1)-w(\alpha) h(\alpha)] d \alpha-v_{0}
$$

where $H$ is the cumulative function of $\alpha ; H(\alpha)=\int_{\underline{\alpha}}^{\alpha} h$, thirdly, since $l$ is non-decreasing and using (8), nonnegativity of $l$ reduces to

$$
l(\underline{\alpha})=\underline{\alpha} v(w(\underline{\alpha}))-v_{0} \geq 0
$$

i.e. $w(\underline{\alpha}) \geq v^{-1}\left(v_{0} / \underline{\alpha}\right)$.

Finally the problem of the firm is:

$$
\max _{w} \Pi(w)=\int_{\underline{\alpha}}^{\bar{\alpha}}[v(w(\alpha))(\alpha h(\alpha)+H(\alpha)-1)-w(\alpha) h(\alpha)] d \alpha
$$

subject to:

- $w$ non-decreasing,

- $w(\underline{\alpha}) \geq \max \left(0, v^{-1}\left(v_{0} / \underline{\alpha}\right)\right)$.

In what follows we will denote by $\underline{w}:=\max \left(0, v^{-1}\left(v_{0} / \underline{\alpha}\right)\right)$ the lower bound on $w$. 


\section{Properties of the optimal labor contract}

In this section, we shortly study the optimal wage function and show that bunching always occurs. In other words, it exists some particular interval of values of $\alpha$ for which fixedwages optimally arise since it is always optimal for the firm not to discriminate among workers on this interval.

Existence and uniqueness of an optimal wage function $w^{*}$ is standard, characterization of $w^{*}$ follows from the ironing procedure of Mussa and Rosen (1978).

Let $w_{0}$ be the solution of the problem obtained from the program of the firm when omitting the monotonicity constraint; clearly $w_{0}$ can be computed analytically:

$$
w_{0}(\alpha)=\left\{\begin{array}{l}
\left(v^{\prime}\right)^{-1}(\psi(\alpha)) \text { if } \alpha h(\alpha)+H(\alpha)>1 \text { and } \psi(\alpha) \leq v^{\prime}(\underline{w}) \\
\underline{w} \text { otherwise }
\end{array}\right.
$$

where $\psi$ is defined by ${ }^{5}$ :

$$
\psi(\alpha):=\frac{h(\alpha)}{\alpha h(\alpha)+H(\alpha)-1}
$$

There exists indeed 2 kinds of sub-intervals of types $\alpha$ :

- those on which there is strict discrimination $d w^{*}>0$; on such intervals, $w^{*}=w_{0}$,

- those on which $w^{*}$ is constant. Such intervals are called bunches. On a bunch, all the agents are offered the same contract for it would be too costly to discriminate among them.

For details of characterization of the solution and first order conditions see Mussa and Rosen (1978) in particular note that $w^{*}$ is continuous with respect to types.

What is specific to our model is that bunching always occurs. This actually follows from the unidimensional reduction of the problem.

Proposition 2 Bunching always occurs; $w^{*}$ cannot be increasing on the whole set of types $[\underline{\alpha}, \bar{\alpha}]$. More precisely, $w^{*}$ is constant and equals $\underline{w}$ for $\alpha$ close to $\underline{\alpha}$.

We have provided a first explanation for both wage differentials and fixed-wages effects via the substitution property. Fixed-wages also result in bunching at the optimum as established in Proposition 2. It is well known that multi-dimensional screening problems

\footnotetext{
${ }^{5}$ Note that $\psi(\alpha)$ is not defined if $\alpha h(\alpha)+H(\alpha)=1$ but in this case $w_{0}(\alpha)=\underline{w}$. Note also that on the set of $\alpha$ 's such that $\psi(\alpha) \geq 0, w_{0}(\alpha)$ is a non-increasing function of $\psi(\alpha)$.
} 
very often lead to bunching (Choné-Rochet, 1998) whereas it is not systematic in onedimensional problems à la Mussa and Rosen. It is interesting to remark that the systematic bunching phenomenon stated in Proposition 2 follows from the two-dimensional nature of the initial problem.

\section{Conclusion}

This note studies optimal labor contracting for a monopsonistic employer confronted with a population of employees who differ by two privately observed characteristics : productivity and subjective discount factor. The motivation is to address a stylized fact in the empirical literature on labor-contracting : productivity differentials only explain a fraction of wage differentials. In our model, the marginal rate of substitution between output and wage depends on both productivity and time preference. We solve this two-dimensional adverse selection problem in a special case (consumption only takes place at period 2) by transforming it into a one-dimensional model; the reduction technique used here can probably be adapted to other contexts of interest. This reduction captures substitution between the two parameters and yields a first explanation to both wage differentials and fixed-wages. Moreover, we prove that in this one-dimensional model bunching occurs at the optimum, which gives a second explanation to fixed-wages. Introducing heterogeneity of workers' time preferences is a reasonable way to explain why productivity differentials are not sufficient to explain wage differentials. Our model is a first step in analyzing a more general line of inquiry : to what degree do workers trade-off time considerations versus ability considerations, and to what extent does it affect optimal labor contracting design.

As mentioned in Paragraph 2.1, another case can be solved. Instead of assuming that consumption only takes place in period 2, one can assume that static preferences are represented by a CARA function. In that case, which is studied in Carlier and Gaumont (2000), the reduction technique still applies and characterization of the optimal wage is very similar : there exists an exhaustive statistic which also leads to a one-dimensional formulation.

Both resolutions prevent any use of two-dimensional contracts. There is no general method to solve almost explicitly the general problem where workers' preferences are given by expression (3). However there is scope for future line of researches. It seems natural for instance to consider that workers differ with respect to other characteristics. 


\section{Appendix}

\section{Density of $\alpha$ :}

The density of $\alpha, h$, can easily be computed and is given by:

$$
h(\alpha)=\int_{\underline{\theta}(\alpha)}^{\bar{\theta}(\alpha)} \frac{1}{\theta} \cdot f(\theta, \alpha / \theta) d \theta .
$$

where $\underline{\theta}(\alpha)$ and $\bar{\theta}(\alpha)$ are given by:

$$
\underline{\theta}(\alpha)=\left\{\begin{array}{l}
\underline{\theta} \text { if } \alpha \leq \alpha_{1} \\
\alpha / \bar{\delta} \text { otherwise }
\end{array} \quad, \bar{\theta}(\alpha)=\left\{\begin{array}{l}
\bar{\theta} \text { if } \alpha \geq \alpha_{2} \\
\alpha / \underline{\delta} \text { otherwise }
\end{array}, \alpha_{1}:=\underline{\theta} \bar{\delta}, \alpha_{2}:=\bar{\theta} \underline{\delta}\right.\right.
$$

Note then that $h$ is continuous and $h(\underline{\alpha})=h(\bar{\alpha})=0$ for $\underline{\theta}(\bar{\alpha})=\bar{\theta}$ and $\bar{\theta}(\underline{\alpha})=\underline{\theta}$. From (12), $h$ is positive in $[\underline{\alpha}, \bar{\alpha}]$ since $f>0$ and a straightforward computation shows that $h$ is decreasing with $h^{\prime}<c<0$ for $\alpha$ sufficiently close to $\bar{\alpha}$.

\section{Proof of Proposition 2:}

Suppose that $w^{*}$ is increasing, the ironing procedure implies that $w^{*}=w_{0}$ so that $w_{0}$ is increasing, where $w_{0}$ is defined in (10). Note that for $\alpha$ close to $\underline{\alpha}$, the function $g(\alpha):=$ $\alpha h(\alpha)+H(\alpha)-1$ is strictly negative so that $w_{\mathbf{0}}$ is constant and equals $\underline{w}$ on a neighbourhood of $\underline{\alpha}$. We get a contradiction which actually proves that bunching occurs at the bottom of the distribution: $w^{*}=\underline{w}$ on a neighbourhood of $\underline{\alpha}$. 


\section{References}

Abowd J.M., Ashenfelter O. (1981) Anticipated Unemployment, Temporary Layoffs and Compensating Wage Differentials. Studies in Labor Markets, Chicago: University of Chicago Press, pp. 141-170.

Azariadis C. (1983) Employment with asymetric information. Quarterly Journal of Economics $98,157-172$.

Carlier G., Gaumont D. (2000) Labor Contracts with two-dimensional adverse selection, working paper, CEREMADE.

Ham J.C. (1982) Estimation of a labor Supply Model with Censoring Due to Unemployment and Underemployment. Review of Economic Studies 49, 333-354.

Killingsworth M. (1988) Labor Supply. Cambridge University Press, Cambridge Surveys of Economic Literature, pp. 61-65.

Krueger A., Summers L.H. (1988) Efficiency Wages and the Inter-Industry Wage Structure. Econometrica 56, 259-293.

Malcomson J. (1981) Unemployment and Efficiency Wages Hypothesis. Economic Journal $91,848-866$.

Mussa M., Rosen S. (1978) Monopoly Quality Product. Journal of Economic Theory 18, 301-317.

Myerson R.B. (1979) Incentive Compatibility and the Bargaining Problem. Econometrica $47,61-74$.

Polemarchakis H., Weiss A. (1978) Fixed-Wages, Layoffs, Unemployment Compensation and Welfare. American Economic Review 68, 909-917.

Spence M. (1974) Market Signalling. Cambridge Massachussets Harvard University Press.

Weiss A. (1980) Job Queues and Layoffs in Labor Market with Flexible Wages. Journal of Political Economy 88, 526-538. 